\title{
The enhancement of tumor radioresponse by combined treatment with cepharanthine is accompanied by the inhibition of DNA damage repair and the induction of apoptosis in oral squamous cell carcinoma
}

\author{
TOYOKO HARADA, KOJI HARADA and YOSHIYA UEYAMA
}

Department of Oral and Maxillofacial Surgery, Yamaguchi University Graduate School of Medicine, Ube, Yamaguchi, Japan

Received January 13, 2012; Accepted March 28, 2012

DOI: 10.3892/ijo.2012.1501

\begin{abstract}
In the present study, we investigated whether treatment with cepharanthine, a biscoclaurine alkaloid extracted from Stephania cepharantha improves the response to radiotherapy in the oral squamous cell carcinoma (OSCC) cell lines, HSC2, HSC 3 and HSC4. We examined the potential mechanisms that may contribute to the enhanced radiation response induced by cepharanthine. Growth inhibition was observed in vitro with radiation or cepharanthine. A co-operative antiproliferative effect was obtained when cancer cells were treated with cepharanthine followed by radiation. Cepharanthine also promoted the mitotic death of 3 cell lines by radiation. The results from DNA damage repair analysis in the cultured OSCC cells demonstrated that cepharanthine had a strong inhibitory effect on DNA double-strand break (DSB) repair after radiation. The combined treatment of cepharanthine and radiation led to an increase in the sub-G1 peak as shown by flow cytometry, and markedly induced apoptosis through the activation of caspase-3. Tumor xenograft studies demonstrated that the combination of cepharanthine and radiation caused growth inhibition and tumor regression of OSCC tumors in athymic mice; tumor volume was reduced from 765.7 to $226.3 \mathrm{~mm}^{3}$ in HSC 2 cells $(\mathrm{p}<0.01), 391.6$ to $43.7 \mathrm{~mm}^{3}$ in HSC3 ( $\left.<<0.01\right)$, and from 572.6 to $174.2 \mathrm{~mm}^{3}$ in HSC4 cells ( $\mathrm{p}<0.01$ ). In addition, combined therapy markedly increased tumor cell apoptosis. Overall, we conclude that cepharanthine enhances tumor radioresponse by multiple mechanisms that may involve the induction of apoptosis and the inhibition of DNA DSB repair after exposure to radiation.
\end{abstract}

Correspondence to: Professor Yoshiya Ueyama, Department of Oral and Maxillofacial Surgery, Yamaguchi University Graduate School of Medicine, 1-1-1 Minamikogushi, Ube, Yamaguchi 755-8505, Japan E-mail: uyoshiya@yamaguchi-u.ac.jp

Key words: radiation, cepharanthine, DNA double-strand break repair, oral squamous cell carcinoma

\section{Introduction}

The incidence of oral squamous cell carcinoma (OSCC) is increasing gradually, and approximately 300,000 new cases of oral cancer are estimated worldwide each year (1-3). OSCC is the most common malignant neoplasm of the oral cavity and represents approximately $90 \%$ of all oral malignancies (4). Radiotherapy as well as surgery have been used for the curative treatment of OSCC. In particular, concurrent chemoradiotherapy has mainly been used for patients with OSCC to preserve oral function. Some patients respond poorly to radiation even when radiotherapy is combined with anticancer agents that possess radiosensitizing effects. This has led to the development of radiosensitizing drugs with relatively few side-effects.

Cepharanthine is one of the biscoclaurine alkaloids extracted from Stephania cepharantha Hayata (5), which has been widely used in Japan for the treatment of a number of acute and chronic diseases, such as bronchial asthma, alopecia areata and leukopenia during radiation therapy or anticancer treatment (6). Additionally, it has been reported that cepharanthine exerts antitumor effects by increasing the immunological competence of the host $(7,8)$. It has also been reported that cepharanthine increases the intracellular accumulation of the anticancer drug, adriamycin, by inhibiting its efflux from tumor tissue (9), while reducing its intracellular accumulation in normal tissue (10). Doxorubicin-resistance may be circumvented by using cepharanthine $(11,12)$. Therefore, cepharanthine may be useful in cancer chemotherapy. In addition, cepharanthine has been shown to exert its antitumor effect by inducing apoptosis in a mouse leukemia cell line (13), an adenosquamous cell carcinoma cell line (14) and an OSCC cell line (15). Recently, we reported on the anti-angiogenic effect of cepharanthine (16), and the combined effects of cepharanthine and the oral 5-fluorouracil pro-drug, S-1, against OSCC (17). However, little is known about the combined effects of cepharanthine and radiation against OSCC.

Radiation induces DNA double-strand breaks (DSBs) in irradiated cells, which may lead to cell killing or tumorigenesis if the DSBs are not repaired properly. The timely and precise repair of DSBs are essential for genome maintenance. Cells have 2 major pathways to repair DSBs: non-homologous endjoining (NHEJ) and homologous recombination (HR) (18). 
NHEJ involves 4 core factors: $\mathrm{Ku}$ serves as a DSB sensor, DNA-PKcs is a protein kinase, XRCC4/LigIV is a DNA ligase and XLF bridges the DSB sensing and ligation steps. These factors assemble on DSBs in a step-by-step manner from $\mathrm{Ku}$ to XLF. XLF has been identified as a new essential factor of NHEJ (19-21). On the contrary, a number of proteins (Nbs1-Mre11-Rad50, BRCA1 and Rad51) exhibit local accumulation after DSB induction in the HR pathway. Rad51 is thought to be a major protein in HR (22). The inhibition of DNA damage repair may also enhance tumor radiosensitivity.

In the present study, we examined the anti-proliferative and apoptosis inducing activity of cepharanthine in combination with radiation in the 3 OSCC cell lines, HSC2, HSC 3 and HSC4. The response of OSCC xenografts in athymic mice to the combined treatment with radiation and cepharanthine was also examined. Furthermore, we investigated various potential mechanisms by which cepharanthine may enhance tumor radiosensitivity.

\section{Materials and methods}

Cell lines and culture. The HSC2, HSC3 and HSC4 cell lines were purchased from Cell Bank, RIKEN BioResource Center (Ibaraki, Japan). Cells were cultured in Dulbe used for normalization of western blot analysis cco's modified Eagle's medium (DMEM) (Sigma-Aldrich, St. Louis, MO, USA) supplemented with $10 \%$ fetal bovine serum (FBS) (Invitrogen, Carlsbad, CA, USA), $100 \mu \mathrm{g} / \mathrm{ml}$ streptomycin, $100 \mathrm{U} / \mathrm{ml}$ penicillin (Invitrogen) in a humidified atmosphere containing $5 \% \mathrm{CO}_{2}$.

Reagent and radiation treatments. For the in vitro assay, cepharanthine was provided by Kaken Shoyaku Co. Ltd. (Tokyo, Japan). The drug was easily dissolved in complete culture medium. Cells on the dish were irradiated with 0-20 Gy using an X-ray irradiator (MBR-1505R2, $150 \mathrm{kV}, 5 \mathrm{~mA}$, filter: $1.0 \mathrm{~mm}$ aluminum; Hitachi Medico, Tokyo, Japan).

In vitro cell growth assay. Cells $\left(5 \times 10^{3}\right.$ cells per well) were seeded on 96-well plates (Becton-Dickinson Labware, Franklin Lakes, NJ, USA) in DMEM supplemented with $10 \%$ FBS. Twenty-four hours later, the cells were either treated with cepharanthine $(0-20 \mu \mathrm{g} / \mathrm{ml})$, or were exposed to X-ray irradiation (0-20 Gy), or both. After 48 h, 3-(4, 5-dimethylthiazol- 2-yl)-2, 5-diphenyltetrazolium bromide (MTT) was added to each well $(25 \mu \mathrm{l} /$ well) and incubated for $4 \mathrm{~h}$. The blue dye absorbed by the cells was dissolved in dimethyl sulfoxide (100 $\mu \mathrm{l} /$ well), and the absorbance was measured with a spectrophotometer (BioRad Laboratories, Hercules, CA, USA) at $490 \mathrm{~nm}$. All assays were run in triplicate.

Clonogenic survival assay. Log-phase cells were trypsinised, counted, and plated in triplicate per data point into 6-well plates. Cells were treated with cepharanthine $(5 \mu \mathrm{g} / \mathrm{ml})$, incubated at $37^{\circ} \mathrm{C}$ for $1 \mathrm{~h}$ before irradiation with X-rays (3, 6, 9, 12, $\left.15 \mathrm{~Gy}\right)$, then returned to $37^{\circ} \mathrm{C}$ for 9 days after the medium was changed with 10\% FBS DMEM. Colonies were fixed with 3:1 methanol/acetic acid and stained with hematoxylin (Muto Chemicals, Tokyo, Japan). Colonies were counted with the naked eye, with a cut-off of 50 viable cells. The surviving fraction (SF) was calculated as mean colonies/(cells inoculated x plating efficiency). Experiments were repeated at least 3 times. Clonogenic survival curves were then plotted.

Western blot analysis. The control- and cepharanthine $(5 \mu \mathrm{g} / \mathrm{ml}$, $1 \mathrm{~h}$ )-treated cells were irradiated (5 Gy). After $30 \mathrm{~min}$ or 48 -h incubation, cells were lysed with RIPA buffer (Thermo Scientific, Rockford, IL, USA). Whole cell lysates were subjected to electrophoresis on $10 \%$ SDS-polyacrylamide gels, and then transferred to PVDF membranes. The membranes were incubated with anti-Rad51 rabbit polyclonal, anti-Ku86 rabbit polyclonal, anti$\mathrm{Ku} 70$ mouse monoclonal, anti-DNA-PKcs mouse monoclonal, anti-Rad50 mouse monoclonal, anti-XRCC4 rabbit polyclonal, anti-XLF rabbit polyclonal and anti-caspase-3 mouse monoclonal antibodies (all from Santa Cruz Biotechnology Inc., Santa Cruz, CA, USA). The antibodies were detected using a chromogenic immunodetection system (WesternBreeze; Invitrogen) according to the manufacturer's instructions. Anti- $\alpha$-tubulin monoclonal antibody (Santa Cruz) was used for the normalization of the proteins from western blot analysis.

Flow cytometry analysis. The control and cepharanthine ( $5 \mu \mathrm{g} / \mathrm{ml}, 1 \mathrm{~h})$-treated cells were irradiated (5 Gy). After 48-h incubation, the attached and floating cells were collected in conical tubes (Becton-Dickinson). The cells were then fixed with $70 \%$ ethanol and washed with PBS. After treatment with $100 \mu \mathrm{g} / \mathrm{ml}$ of RNase A (Sigma-Aldrich), the cells were stained with $40 \mu \mathrm{g} / \mathrm{ml}$ propidium iodine (Molecular Probes, Eugene, OR, USA), and the cell cycle was analysed by a digital flow cytometry system (Cytomics FC500; Beckman Coulter, Miami, FL, USA).

Nude mice and breeding. Female athymic nude mice with a CAnN.Cg-Foxnlnu/CrlCrlj genetic background (CLEA Japan Inc., Tokyo, Japan) were purchased at 4 weeks of age and kept under sterile conditions in a pathogen-free environment. The mice were provided with sterile water and food ad libitum and all manipulations were carried out aseptically inside a laminar flow hood. The mice were maintained and handled in accordance with the Guidelines for Animal Experimentation of Yamaguchi University, Ube, Japan.

In vivo tumor growth assay. The effect of combined cepharanthine treatment and radiation exposure was assessed by the inoculation of cells into 5-week-old female athymic nude mice. Cells $\left(1 \times 10^{6}\right)$ were suspended in $0.1 \mathrm{ml}$ of serum-free medium and injected into the subcutaneous tissue of mice (average weight, $15.0 \mathrm{~g}$ ) using a 27-gauge needle. Tumors at the inoculation site were monitored and measured. When the tumors reached $100-150 \mathrm{~mm}^{3}$ in volume, the mice were divided into 4 groups, and treated with cepharanthine and/or exposed to radiation for 3 weeks. Briefly, cepharanthine $(20 \mathrm{mg} / \mathrm{kg})$ was injected into the peritumoral tissue for 3 weeks ( 5 times/week). Tumors on the flanks of mice were irradiated with 1.5 Gy for 3 weeks (5 times/week) using X-ray irradiation (MBR-1505R2, $150 \mathrm{kV}, 5 \mathrm{~mA}$; filter, $1.0 \mathrm{~mm}$ aluminum). The dose rate was $1.18 \mathrm{~Gy} / \mathrm{min}$ at a source-skin distance of $56.5 \mathrm{~cm}$. The nontumor parts of mice were shielded using lead blocks. The mice in the control group also received saline $(200 \mu \mathrm{l})$ by peritumoral injection. The tumors were measured every 2 days and the tumor volumes were calculated. At 21 days, mice were sacri- 
(A)

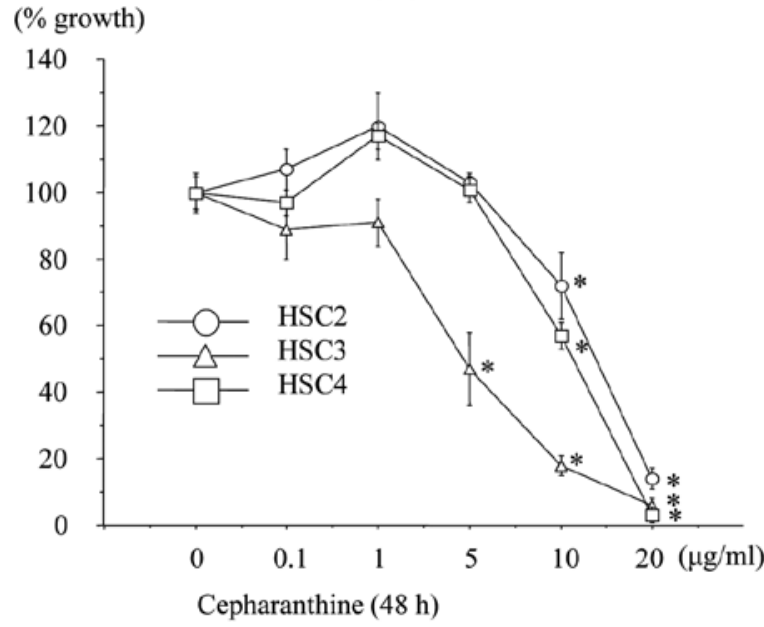

$*: \mathrm{P}<0.01$ when compared with that of control $(0 \mu \mathrm{g} / \mathrm{ml})$ by Mann-Whitney U test
(B)

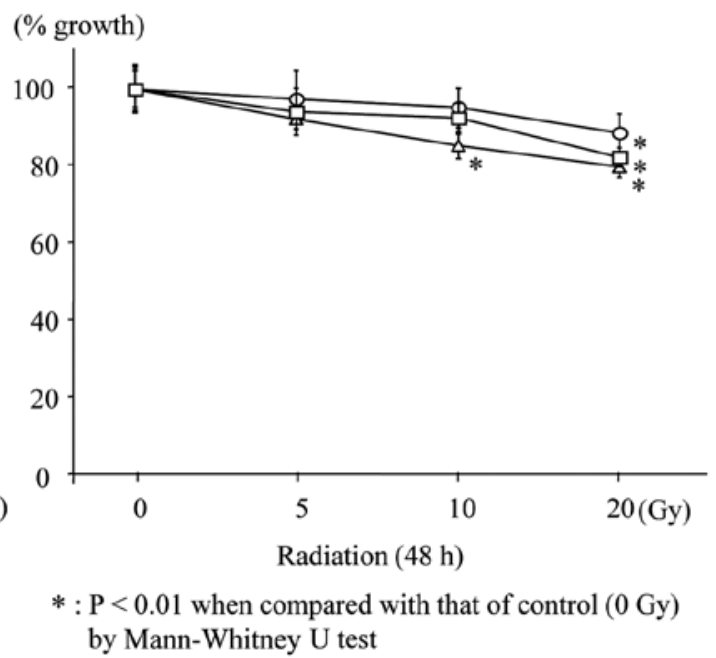

Figure 1. Growth inhibitory effect of cepharanthine and/or radiation on OSCC cells. (A) Cells (5x10 ${ }^{3}$ cells per well) were seeded on 96 -well plates. After 24 h, cells were treated with cepharanthine $(0.1-20 \mu \mathrm{g} / \mathrm{ml})$ alone. After $48 \mathrm{~h}$, in vitro cell growth was evaluated by MTT assay as the percentage growth. (B) Cells $\left(5 \mathrm{x} 10^{3}\right.$ cells per well) were seeded on 96-well plates. After $24 \mathrm{~h}$, cells were exposed to radiation (0-20 Gy) alone. After 48 h, in vitro cell growth was evaluated by MTT assay as the percentage growth. Error bars represent the standard deviation of the mean of 3 independent experiments.

ficed by cervical dislocation and the tumors were dissected out, fixed in neutral-buffered formalin and embedded in paraffin for further study.

Terminal deoxynucleotidyl transferase (TdT)-mediated nick end-labeling (TUNEL) assay. To detect apoptotic cells, the ApopTag Plus peroxidase in situ Apoptosis Detection kit (Intergen Co., Purchase, NY, USA) was used. Paraffin sections of tumor (4- $\mu \mathrm{m}$-thick) were deparaffinized in xylene and rehydrated in decreasing concentrations of ethanol. Tissue sections were incubated in $20 \mu \mathrm{g} / \mathrm{ml}$ proteinase $\mathrm{K}$ (Dako, Glostrup, Denmark) for $15 \mathrm{~min}$. The sections were then rinsed in distilled water, endogenous peroxidase was blocked by incubating the slides in a $3 \%$ hydrogen peroxide solution for $5 \mathrm{~min}$. After being washed with PBS (0.05 M phosphate buffer containing $0.145 \mathrm{M}$ sodium chloride, $\mathrm{pH}$ 7.4), the sections were incubated with equilibration buffer and then TdT enzyme in a humidified chamber at $37^{\circ} \mathrm{C}$ for $60 \mathrm{~min}$. They were subsequently placed into pre-warmed working strength stop wash buffer for $10 \mathrm{~min}$. After being rinsed with PBS, the sections were incubated with anti-digoxigenin-peroxidase conjugate for $30 \mathrm{~min}$. Peroxidase activity in each section was demonstrated by the application of diaminobenzidine (Peroxidase Substrate kit; Vector Laboratories). Hematoxylin was used as the counterstain. At least 1,000 cells were counted under a microscope in several random fields of each section. The number of apoptotic cells was calculated by the number of TUNEL-positive cells divided by the total number of counted cells and the result was expressed as a percentage.

Statistical analysis. Statistical significance was set at $\mathrm{p}<0.05$. Statistical analyses were performed using the StatView software (version 5.0J, SAS Institute Inc., Cary, NC, USA).

\section{Results}

Cepharanthine augments cell growth inhibition by radiation and enhances radiosensitivity in vitro. We examined the effects of cepharanthine and radiation on the viability of OSCC cells. Cell viability was evaluated by MTT assay after $48 \mathrm{~h}$ of treatment with cepharanthine or radiation at various cepharanthine concentrations or radiation doses. HSC3 cell growth decreased at concentrations of 5-20 $\mu \mathrm{g} / \mathrm{ml}$ of cepharanthine, and HSC2 and HSC4 cell growth decreased at $10-20 \mu \mathrm{g} / \mathrm{ml}$ of cepharanthine (Fig. 1A). Radiation inhibited cell growth in a dose-dependent manner. HSC3 cell growth decreased at dose of 10-20 Gy, and HSC2 and HSC4 cell growth decreased at a dose of 20 Gy (Fig. 1B). Briefly, HSC3 cells were more sensitive to cepharanthine or radiation than HSC2 and HSC4 cells. To determine whether the cepharanthine treatment of OSCC cells enhances the radiation effect, OSCC cells were treated with cepharanthine $(5 \mu \mathrm{g} / \mathrm{ml})$ and exposed to radiation (5 Gy). The exposure of OSCC cells to radiation with cepharanthine resulted in a significant growth inhibition when compared to treatment with cepharanthine or radiation alone (Fig. 2). We also examined whether the addition of cepharanthine enhances radiosensitivity (mitotic death by radiation). OSCC cell lines were treated with radiation alone or in combination with cepharanthine $(5 \mu \mathrm{g} / \mathrm{ml})$. The dose of radiation required to give an SF of $10 \%$ for each cell line was decreased $(\mathrm{p}<0.05)$ in the presence of cepharanthine (HSC2, 14.7-9.5 Gy; HSC3, 13.3-9.0 Gy; HSC4, 14.3-9.7 Gy). Thus, the addition of cepharanthine enhanced the radiosensitivity of all cell lines by 1.47 - to 1.55 -fold (Fig. 3 ).

Cepharanthine affects the DNA repair pathway after radiation. To investigate mechanisms by which cepharanthine exerts its radiosensitizing effect on OSCC cells, the expression of 


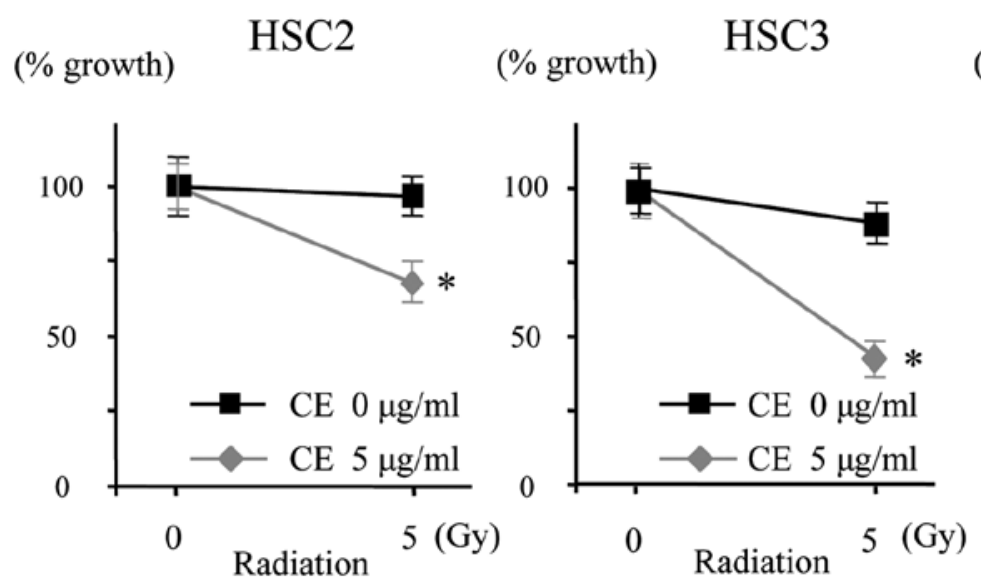

(\% growth) HSC4

*: $\mathrm{P}<0.01$ when compared with that of $\mathrm{CE}$ alone or RT alone by Mann-Whitney U test. $\quad \mathrm{CE}$, Cepharanthine

Figure 2. Combined effects of cepharanthine and radiation on OSCC cells. Cells $\left(5 \times 10^{3}\right.$ cells per well) were seeded on 96 -well plates. After $24 \mathrm{~h}$, cells were exposed to radiation ( $5 \mathrm{~Gy}$ ) after treatment with cepharanthine $(5 \mu \mathrm{g} / \mathrm{ml})$. After $48 \mathrm{~h}$, in vitro cell growth was evaluated by MTT assay as the percentage growth. Error bars represent the standard deviation of the mean of 3 independent experiments.

HSC2

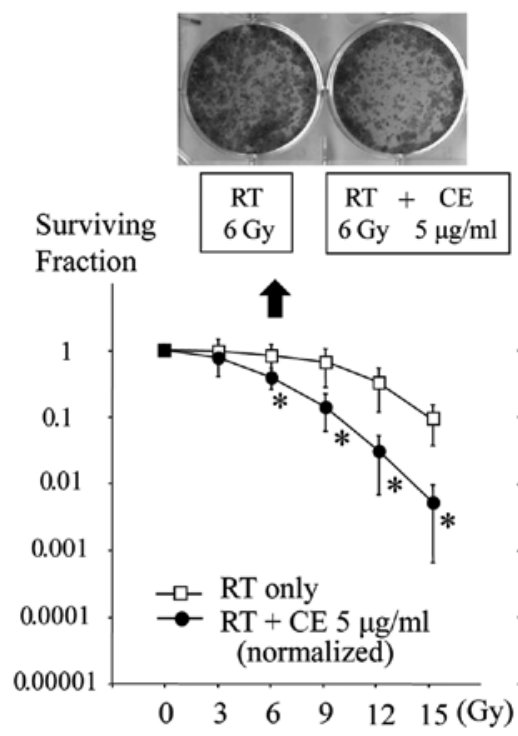

HSC3
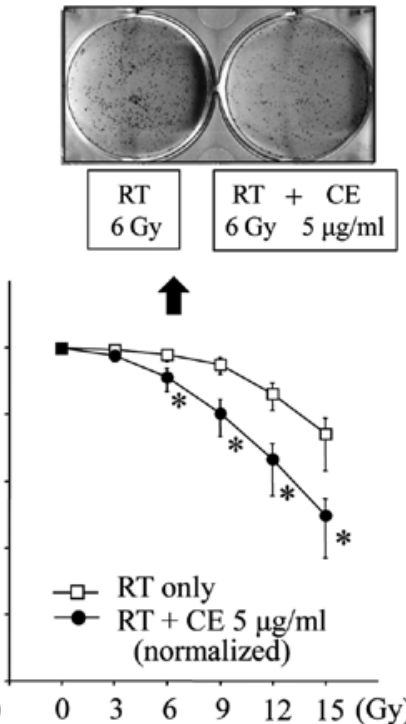

HSC4
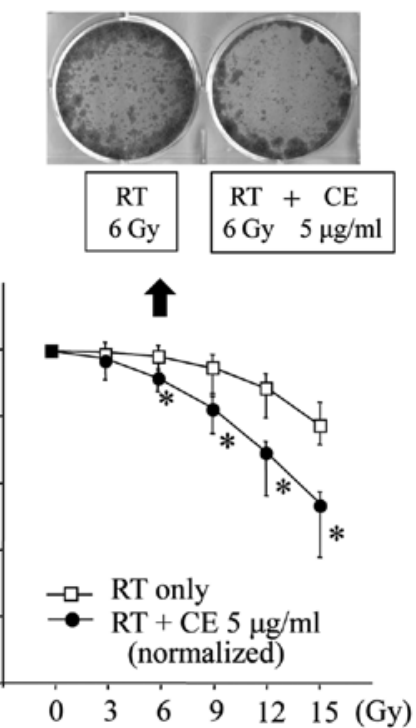

Surviving Fraction $=$ mean colonies $/$ cells inoculated $\times$ plating efficiency

$$
\text { *; }<0.05 \text { (t-test) } \quad \text { RT + CE vs RT alone }
$$

CE, Cepharanthine
RT, Radiation

Figure 3. Clonogenic survival curves of OSCC cells. Attached cells were treated with radiation alone, or cepharanthine (5 $\mu \mathrm{g} / \mathrm{ml}) \mathrm{plus}$ radiation (0-15 Gy). After 9 days, colonies were fixed and stained with hematoxylin. Colonies were counted with the naked eye, with a cut-off of 50 viable cells. The surviving fraction (SF) was calculated. Error bars represent the standard error of the mean of 3 independent experiments. Radiosensitivity improved by 1.47 - to 1.55 -fold with the addition of cepharanthine.

DNA-DSB repair proteins was examined by western blot analysis (Fig. 4). To examine the effect of cepharanthine on the expression of DNA-DSB repair proteins after radiation, OSCC cells were treated with cepharanthine $(5 \mu \mathrm{g} / \mathrm{ml})$ for $1 \mathrm{~h}$ after radiation (5 Gy). The combined treatment with radiation and cepharanthine reduced the levels of Rad51, Ku86, Ku70, DNA-PKcs, Rad50, XRCC4 and XLF in each cell. DNA-DSB repair protein levels were markedly reduced in HSC3 cells when compared to those in HSC2 or HSC4 cells.
Induction of apoptosis by combined treatment of cepharanthine and radiation. We then investigated whether the combined effects of cepharanthine and radiation on in vitro cell growth of OSCC cells is associated with the induction of apoptosis. Therefore, the activation of procaspase-3 was examined after treatment with cepharanthine alone, radiation alone or the combination of both. As shown by western blot analysis, we observed an enhanced activation of procaspase- 3 in the cells treated with cepharanthine in combination with radiation 


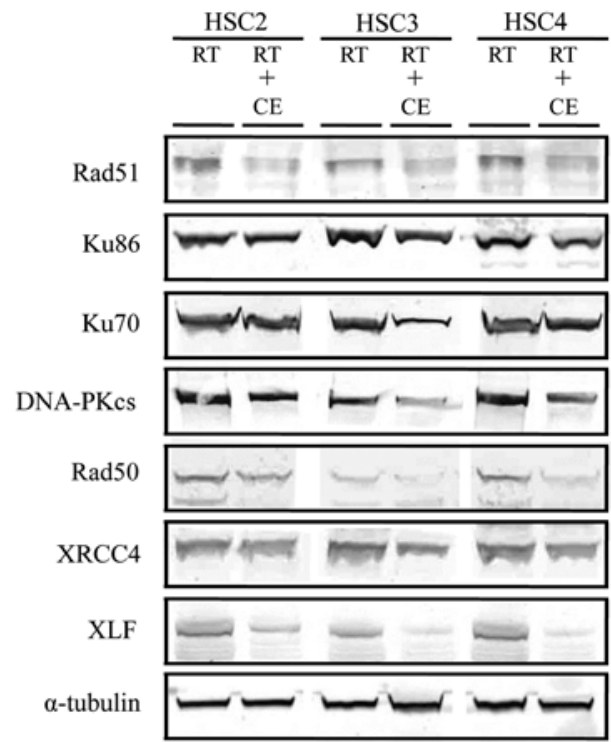

Figure 4. Effect of cepharanthine and radiation on the expression of DNA-DSB repair proteins in OSCC cells. Control and cepharanthine $(5 \mu \mathrm{g} / \mathrm{ml}, 1 \mathrm{~h})-$ treated cells were irradiated (5 Gy). After 30-min incubation, cells were collected and lysed. Whole cell lysates were subjected to electrophoresis on $10 \%$ SDS-polyacrylamide gels, and then transferred to PVDF membranes. The membranes were incubated with the antibodies against DSB repair proteins (Rad51, Ku86, ku70, DNA-PKcs, Rad50, XRCC4 and XLF) as described in Materials and methods. RT, radiotherapy; CE, cepharanthine. when compared to the cells treated with either reagent alone or no treatment (Fig. 5A). Cell cycle changes associated with the combined treatment of cepharanthine and radiation in each cell were analysed using a digital flow cytometry system. We detected a G1 phase arrest in cepharanthine-treated cells and a G2/M phase arrest in radiation-treated cells. A sub-G1 peak was notably high in the cells treated with cepharanthine and radiation, although a sub-G1 peak was observed in the cepharanthine- and radiation-treated cells (Fig. 5B).

The effect of combined therapy with cepharanthine and radiation on tumor growth in vivo in nude mice. On the basis of the above in vitro findings, we examined the effects of cepharanthine and radiation on in vivo tumor growth. Each cell was injected into the backs of nude mice subcutaneously, and the treatment with cepharanthine and/or radiation commenced when the tumor volume reached $100-200 \mathrm{~mm}^{3}$. As shown in Fig. 6A, treatment with radiation or cepharanthine alone produced modest inhibition of tumor growth in HSC2, HSC3 and HSC4 cell xenografts, and that combined treatment with radiation and cepharanthine produced a marked inhibition of tumor growth in all mice.

Systemic therapy with cepharanthine alone, radiation alone and cepharanthine in combination with radiation resulted in growth inhibition and tumor regression; tumor volume was

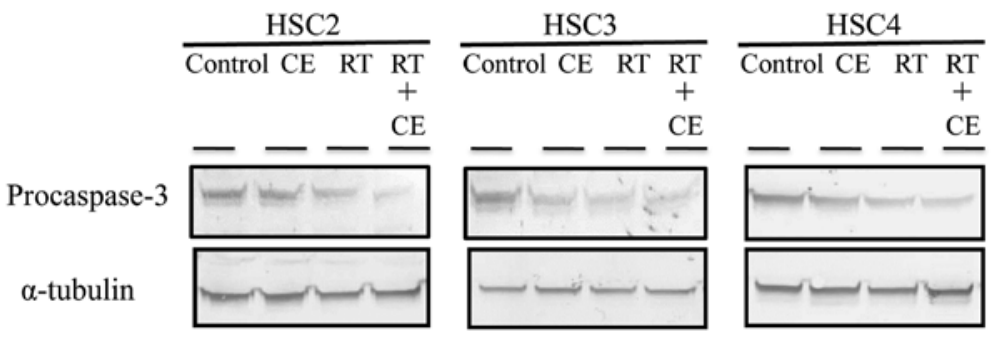

(B)

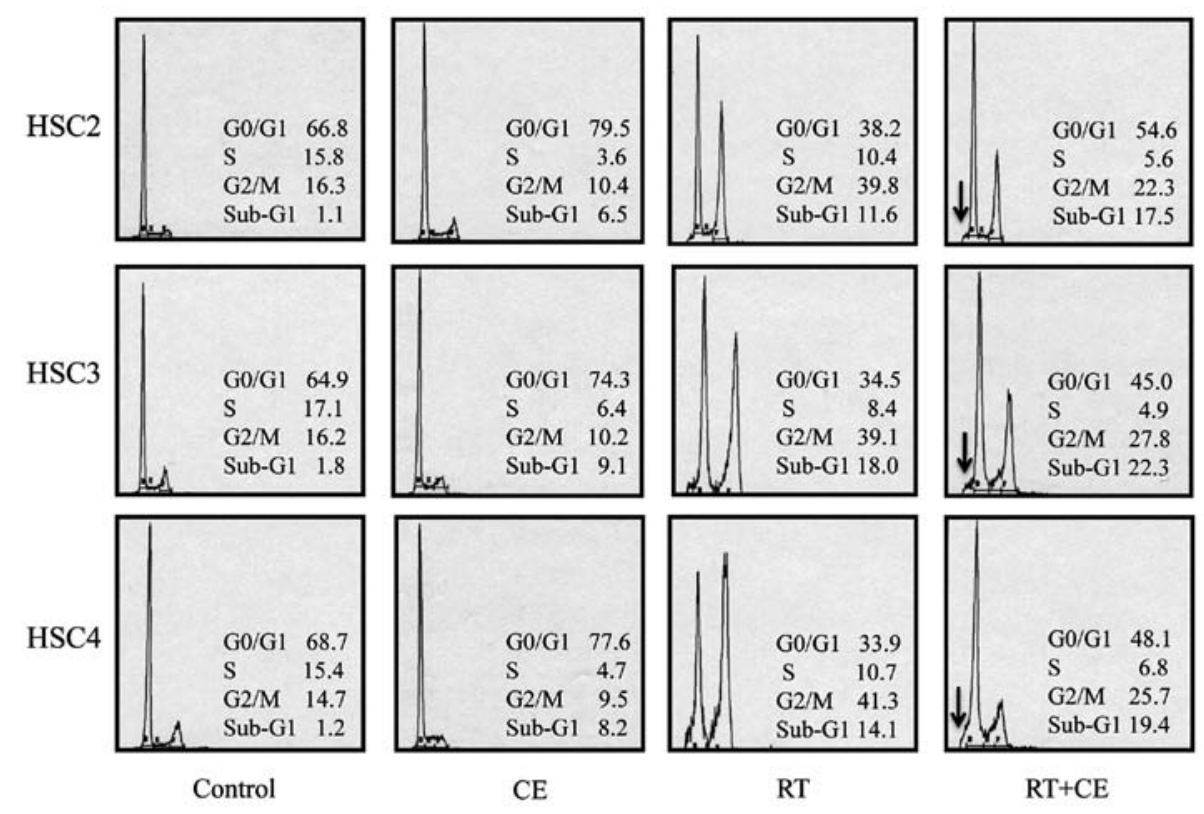

Figure 5. Induction of apoptosis by the combined treatment of cepharanthine and radiation in OSCC cells. Control and cepharanthine ( $5 \mu \mathrm{g} / \mathrm{ml}, 1 \mathrm{~h})$-treated cells were irradiated (5 Gy). After 48-h incubation, cells were collected. (A) Whole cell lysates were subjected to electrophoresis on 10\% SDS-polyacrylamide gels, and then transferred to PVDF membranes. The membranes were incubated with the antibodies against procaspase-3 as described in Materials and methods. (B) Cells were fixed with $70 \%$ ethanol and washed with PBS. After treatment with RNase A, the cells were stained with propidium iodine, and the cell cycle and sub-G1 peak (arrow) were analysed by a digital flow cytometry system. RT, radiotherapy; CE, cepharanthine. 


\section{(A)}

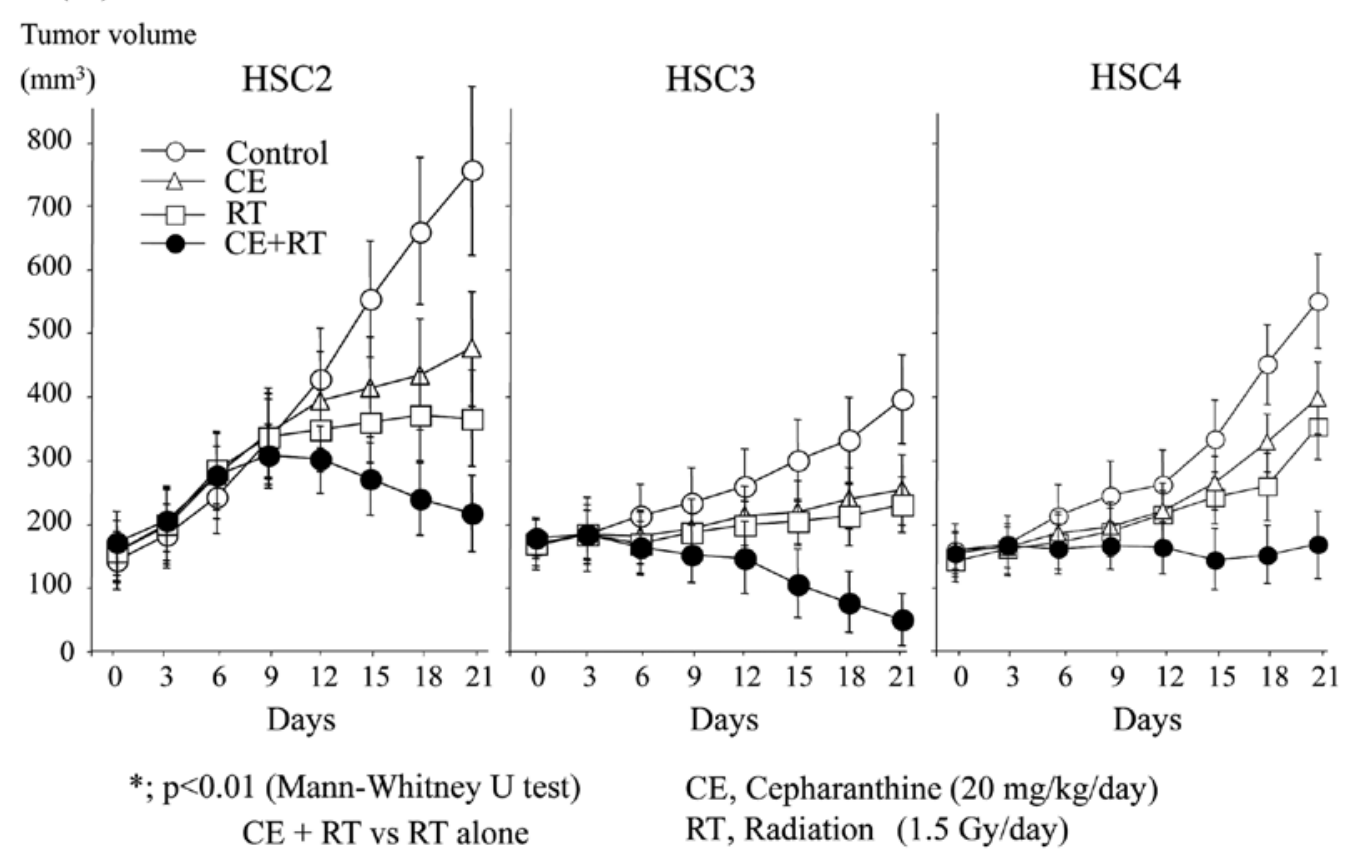

(B)

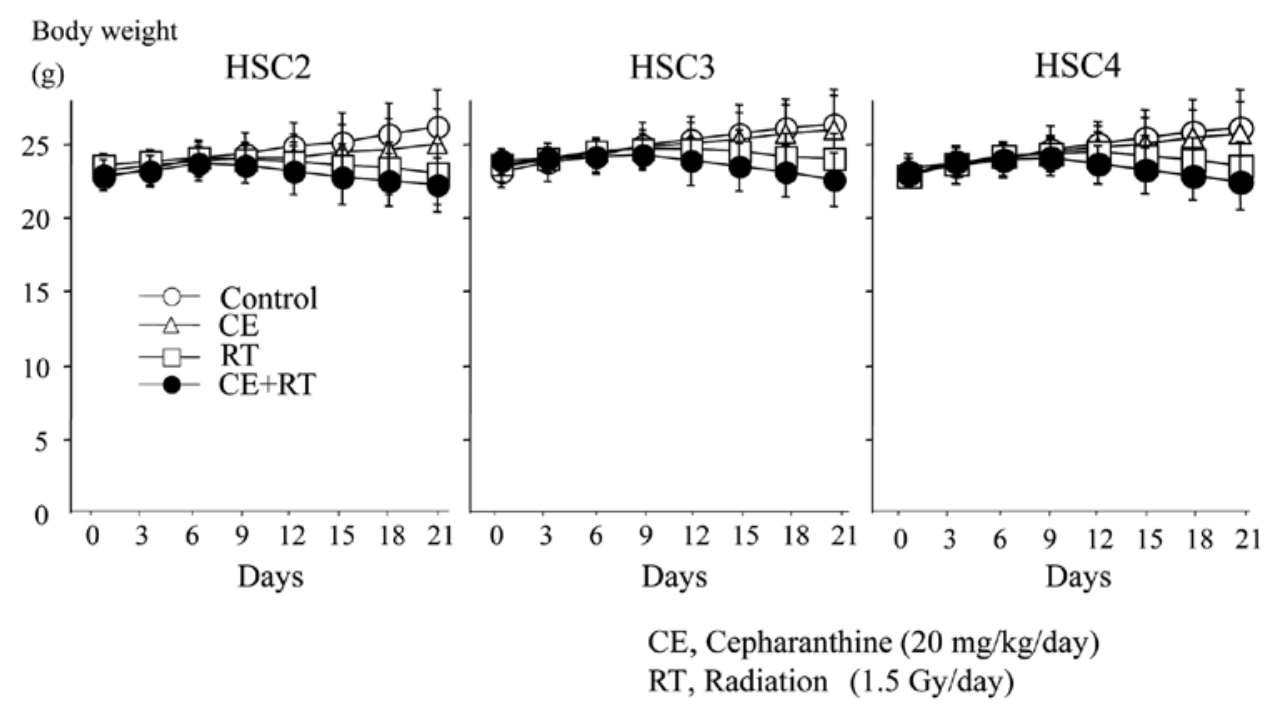

Figure 6. Antitumor activity of cepharanthine in combination with radiation in HSC2, HSC 3 and HSC4 cell xenografts. Cells (1x10 6 ) were inoculated into the backs of nude mice. When the tumors reached $100-200 \mathrm{~mm}^{3}$ in volume, they were treated with cepharanthine and/or exposed to radiation for 3 weeks. (A) Tumor volume and (B) body weight were measured every 2 days. Error bars represent the standard error of the mean from 3 independent experiments. Each experiment contained 5 mice per treatment group. RT, radiotherapy; CE, cepharanthine.

reduced from 765.7 in the controls to $475.3,371.7$ and $226.3 \mathrm{~mm}^{3}$ in the HSC2 cell xenografts $(\mathrm{p}<0.01)$ and from 391.6 in the controls to $248.91,223.6$ and $43.7 \mathrm{~mm}^{3}$ in the HSC 3 cell xenografts $(\mathrm{p}<0.01$ ), and from 572.6 in the controls to $392.4,367.0$ and $174.2 \mathrm{~mm}^{3}$ in the HSC4 cell xenografts $(\mathrm{p}<0.01)$. During the experimental period, no loss of body weight was observed in the mice treated with the combined therapy (Fig. 6B). Furthermore, the induction of apoptosis was examined in all OSCC tumor xenografts. Apoptotic cells were examined by TUNEL assay. The apoptotic cells in the HSC2 tumors are shown in Fig. 7A. The TUNEL-positive cells were significantly increased in each tumor treated with cepharanthine and radiation when compared to each tumor treated with either agent alone (Fig. 7B).

\section{Discussion}

In this study, we focused on the various biological and pharmacological activities of cepharanthine. We report that cepharanthine suppresses tumor growth by inducing cell cycle arrest through the downregulation of cyclin E and the upregulation of p27 (15) or p21 (14), and by inducing apoptotic effects through the activation of caspase- 9 and caspase-3. Recently, we reported on the 
(A)

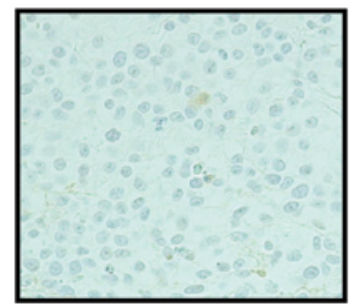

Control

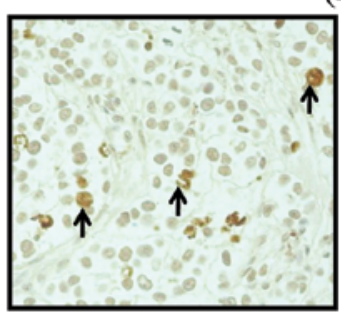

$\mathrm{CE}: 20 \mathrm{mg} / \mathrm{kg} / \mathrm{day}$

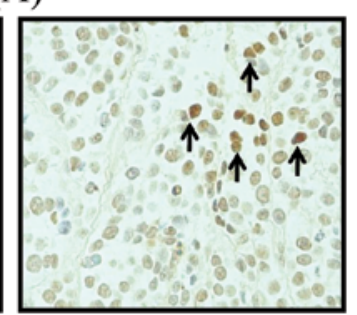

RT: $1.5 \mathrm{~Gy} / \mathrm{day}$

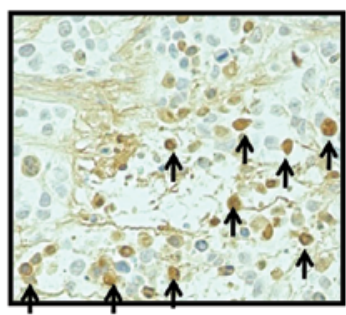

CE+RT

$\uparrow$ Apoptotic cell

(B)

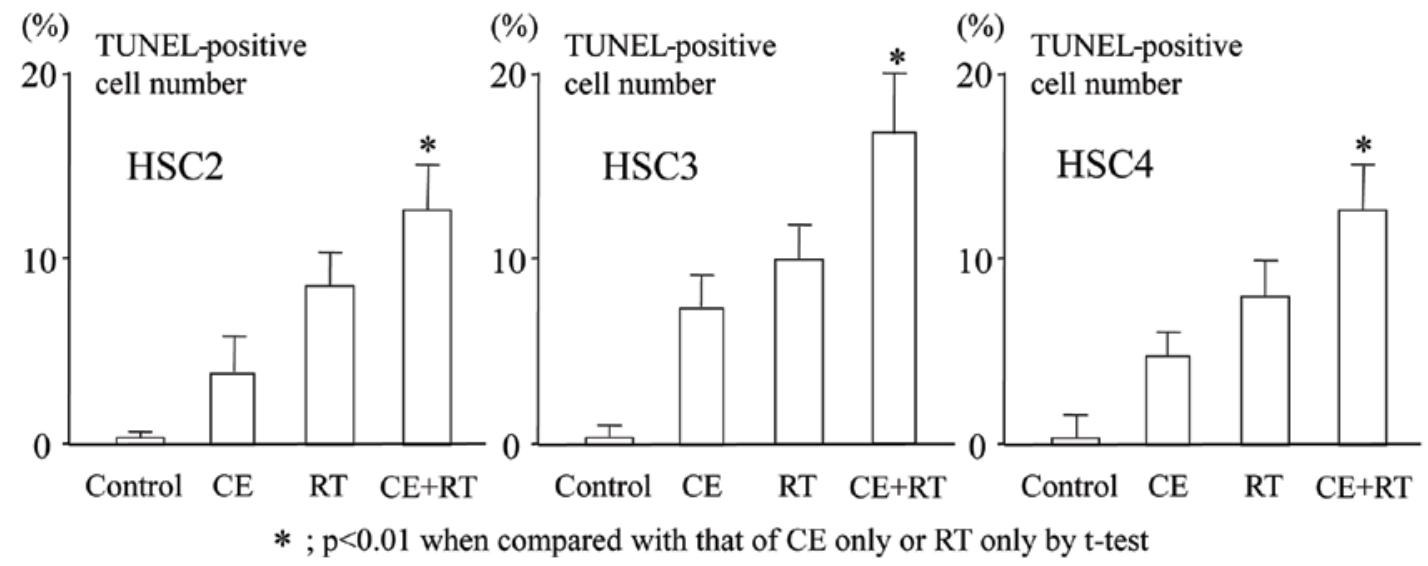

Figure 7. Effect of cepharanthine and/or radiation on the induction of apoptosis in HSC2, HSC 3 and HSC4 tumors. The tumors were dissected out, fixed in neutral-buffered formalin and embedded in paraffin at 21 days of in vivo tumor growth assay. Paraffin sections of tumors (4- $\mu \mathrm{m}$-thick) were made, and apoptotic cells were detected by TUNEL assay in each tumor as described in Material and methods. The number of apoptotic cells was calculated as the number of TUNEL-positive cells divided by the total number of counted cells, and the result was expressed as a percentage. (A) TUNEL-positive cells in HSC2 tumor. (B) TUNEL-positive cell number in each tumor. RT, radiotherapy; CE, cepharanthine.

anti-angiogenic effect of cepharanthine (16), and the combined effects of cepharanthine and the oral 5-fluorouracil pro-drug, S-1, against OSCC (17). Other studies have also reported that cepharanthine exerts anti-tumor effects by inhibiting the activity of P-glycoprotein $(23,24)$, and regulating the expression of Bcl-2 and Bax protein (25,26). From these perspectives, cepharanthine should be useful for anticancer treatment. However, little is known about the detailed mechanisms of the antitumor activity of cepharanthine in solid tumors, including OSCC. Many patients with advanced OSCC desire a treatment other than surgery or functional preservation therapy. Chemoradiotherapy is then selected as the primary treatment. In this case, cepharanthine is administered during chemo-radiotherapy for the treatment of leukopenia, as well as mucositis. However, little is known about the combined effects of cepharanthine and radiation against OSCC. In this study, we investigated the combined effects of cepharanthine and radiation against OSCC.

Cepharanthine or radiation alone was ineffective against HSC2 and HSC4 cells, while HSC 3 cells were more sensitive to cepharanthine or radiation than HSC2 and HSC4 cells (Fig. 1). The combined effect of cepharanthine and radiation was also observed in in all 3 cell lines (Fig. 2). From these findings, it is evident that cepharanthine can act as a radiosensitiser. We then investigated whether cepharanthine can promote the mitotic death of cancer cells by radiation. The combined treatment of cepharanthine and radiation suppressed the colony forming capacity of the cancer cells, as opposed to radiation alone
(Fig. 3). Cepharanthine may be a safe and useful radiosensitiser as it has little adverse effects clinically. We did not detect any adverse effects, such as loss of body weight in the tumor-bearing nude mice treated with cepharanthine alone (Fig. 6B).

Radiosensitisers are thought to increase the radiosensitivity by inhibiting the repair of radiation-induced DNA damage. We then examined whether cepharanthine can regulate the expression of DNA-DSB repair proteins induced by radiation. The expressions of DSB repair proteins $(\operatorname{Rad} 51, \mathrm{Ku} 86, \mathrm{Ku} 70$, DNA-PKcs, Rad50, XRCC4 and XLF) were decreased in OSCC cells treated with cepharanthine and radiation, compared to the cells treated with radiation alone. Briefly, the expressions of DNA-DSB repair proteins related to NHEJ and HR were suppressed by the combined treatment of cepharanthine and radiation. Of note, the expressions of DNA-DSB repair proteins (Ku70, DNA-PKcs and XECC4) were markedly suppressed in the HSC 3 cells as opposed to the HSC2 and HSC 4 cells. HSC 3 cells may have been more sensitive to cepharanthine and radiation than $\mathrm{HSC} 2$ and $\mathrm{HSC} 4$ cells, as the DNA-DSB repair system was inhibited effectively in the HSC3 cells (Fig. 4). The combined treatment of cepharanthine and radiation not only inhibited the repair of radiation-induced DNA damage, but also enhanced radiation-induced apoptosis in OSCC cells (Fig. 5).

Similarly, the combination of cepharanthine and radiation was more effective against human tumor xenografts than either agent separately (Fig. 6A). Also, the main mechanism of antitumor activity was thought to be the augmentation of apoptosis 
(Fig. 7). Moreover, adverse effects including loss of body weight were not detected in the tumor-bearing nude mice treated with cepharanthine and radiation (Fig. 6B). These findings suggest that the combination therapy of cepharanthine and radiation is safe for the body. Suitable doses of cepharanthine and radiation for combination therapy must be developed.

In conclusion, cepharanthine enhances the effects of radiation both in vitro and in vivo in human OSCC models. This study also shows that there may be an advantage to using both agents together when compared to either agent separately. These preclinical data show that cepharanthine may have clinical utility in combination with radiotherapy against OSCC.

\section{Acknowledgements}

This study was supported in part by a Grant-in-Aid from the Japanese Ministry of Education, Science and Culture.

\section{References}

1. Rautava J, Luukkaa M, Heikinheimo K, Alin J, Grenman R and Happonen RP: Squamous cell carcinomas arising from different types of oral epithelia differ in their tumor and patient characteristics and survival. Oral Oncol 43: 911-919, 2007.

2. Funk GF, Karnell LH, Robinson RA, Zhen WK, Trask DK and Hoffman HT: Presentation, treatment, and outcome of oral cavity cancer: a national cancer data base report. Head Neck 24: 165-180, 2002

3. Mehrotra R, Singh MK, Pandya S and Singh M: The use of an oral brush biopsy without computer-assisted anaylsis in the ora lesions: a study of 94 patients. Oral Surg Oral Med Oral Pathol Oral Radiol Endod 106: 246-253, 2008.

4. Lawoyin JO, Lawoyin DO and Aderinokun G: Intra-oral squamous cell carcinoma in Ibadan: a review of 90 cases. Afr J Med Med Sci 26: 187-188, 1997.

5. Tomita M,Fujitani K and Aoyagi Y: Synthesis of dl-cepharanthine. Tetrahedron Lett 13: 1201-1206, 1967.

6. Saito R, Tsuchiya S, Ishizuka T, Fueki N, Ezawa K, Minato K, Nakano H, Takise A, Kurihara M and Fueki R: Clinical effects of cepharanthine (Ceph.) on leukopenia by chemotherapy in lung cancer patients. Nihon Gan Chiryo Gakkai Shi 24: 2587-2593, 1989.

7. Ono M: Antitumor effect of cepharanthine: activation of regional lymph node lymphocytes by intratumoral administration. Clin Immunol 19: 1061-1064, 1987.

8. Morioka S, Ono M, Tanaka N and Orita K: Synergistic activation of rat alveolar macrophages by cepharanthine and OK-432. Gan To Kagaku Ryoho 12: 1470-1475, 1985 (In Japanese).

9. Asaumi J, Kawasaki S, Nishikawa K, Kuroda M and Hiraki Y Effects of hyperthermia and cepharanthine on adriamycin accumulation with change in extracellular $\mathrm{pH}$. Int $\mathrm{J}$ Hyperthermia 11: 27-35, 1995.

10. Nishikawa K, Asaumi J, Kawasaki S, Kuroda M, Takeda Y and Hiraki Y: Influence of cepharanthine on the intracelluar accumulation of adriamycin in normal liver cells and spleen cells of mice in vitro and in vivo. Anticancer Res 17: 3617-22, 1997.

11. Hotta $T$, Tanimura $H$, Yamaue $H$, Iwahashi M, Tani $M$, Tsunoda T, Tamai M, Noguchi K, Mizobata S, Arii K and Terasawa H: Modulation of multidrug resistance by cepharanthine in fresh human gastrointestinal tumor cells. Oncology 54: 153-157, 1997.
12. Hotta T, Tanimura H, Yamaue H, Iwahashi M, Tani M, Tsunoda T, Noguchi K, Mizobata S and Terasawa H: Synergistic effects of tamoxifen and cepharanthine for circumventing the multidrug resistance. Cancer Lett 107: 117-123, 1996.

13. Furusawa S, Wu J, Fujimura T, Nakano S, Nemoto $S$, Takayanagi M, Sasaki K and Takayanagi Y: Cepharanthine inhibits proliferation of cancer cells by inducing apoptosis. Methods Find Exp Clin Pharmacol 20: 87-97, 1998.

14. Harada K, Bando T, Yoshida H and Sato M: Characteristics of antitumour activity of cepharanthin against a human adenosquamous cell carcinoma cell line. Oral Oncol 37: 643-651, 2001.

15. Harada K, Supriatno, Yamamoto S, Kawaguchi S, Yoshida H and Sato M: Cepharanthine exerts antitumor activity on oral squamous cell carcinoma cell lines by induction of p27Kip1. Anticancer Res 23: 1441-1448, 2003.

16. Harada K, Ferdous T, Itashiki Y, Takii M, Mano T, Mori Y and Ueyama Y: Cepharanthine inhibits angiogenesis and tumorigenicity of human oral squamous cell carcinoma cells by suppressing expression of vascular endothelial growth factor and interleukin-8. Int J Oncol 35: 1025-1035, 2009.

17. Harada K, Ferdous T, Itashiki Y, Takii M, Mano T, Mori Y and Ueyama Y: Effects of cepharanthine alone and in combination with fluoropyrimidine anticancer agent, $\mathrm{S}-1$, on tumor growth of human oral squamous cell carcinoma xenografts in nude mice. Anticancer Res 29: 1263-1270, 2009.

18. Burma S, Chen BPC and Chen DJ: Role of non- homologous end joining (NHEJ) in maintaining genomic integrity. DNA Repair (Amst) 5: 1042-1048, 2006

19. Buck D, Malivert L, de Chasseval R, Barraud A, Fondaneche MC, Sanal O, Plebani A, Stephan JL, Hufnagel M, le Deist F, Fischer A, Durandy A, de Villartay JP and Revy P: Cernunnos, a novel nonhomologous end-joining factor, is mutated in human immunodeficiency with microcephaly. Cell 124: 287-299, 2006.

20. Ahnesorg P, Smith P and Jackson SP: XLF interacts with the XRCC4-DNA ligase IV complex to promote DNA nonhomologous end-joining. Cell 124: 301-313, 2006.

21. Callebaut I, Malivert L, Fischer A, et al: Cernunnos interacts with the XRCC4 $x$ DNA-ligase IV complex and is homologous to the yeast nonhomologous end-joining factor Nej1. J Biol Chem 281: 13857-13860, 2006.

22. O'Regan P, Wilson C, Townsend S and Thacker J: XRCC2 is a nuclear RAD51-like protein required for damage-dependent RAD51 focus formation without the need for ATP binding. J Biol Chem 276: 22148-22153, 2001.

23. Nakajima A, Yamamoto Y, Taura K, et al: Beneficial effect of cepharanthine on overcoming drug-resistance of hepatocellular carcinoma. Int J Oncol 24: 635-645, 2004.

24. Ikeda R, Che XF, Yamaguchi T, et al: Cepharanthine potently enhances the sensitivity of anticancer agents in K562 cells. Cancer Sci 96: 372-376, 2005.

25. Biswas KK, Tancharoen S, Sarker KP, Kawahara K, Hashiguchi T and Maruyama I: Cepharanthine triggers apoptosis in a human hepatocellular carcinoma cell line $(\mathrm{HuH}-7)$ through the activation of JNK1/2 and the downregulation of Akt. FEBS Lett 580: 703-710, 2006.

26. Kikukawa Y, Okuno Y, Tatetsu H, et al: Induction of cell cycle arrest and apoptosis in myeloma cells by cepharanthine, a biscoclaurine alkaloid. Int J Oncol 33: 807-814, 2008. 